\title{
Independent Component Analysis for IoT Services Using Machine Learning N NITHYANANDAM
}

M. Tech Scholar, Department of Computer Science and Engineering, Jntuacea, Ananthapuramu, Andhra

Pradesh, India

\section{ABSTRACT}

Article Info

Volume 7, Issue 2

Page Number: 309-316

Publication Issue :

March-April-2021

\section{Article History}

Accepted : 12 April 2021

Published : 17 April 2021
Machine learning is an implementation of Artificial Intelligence (AI) that allows devices to learn and develop independently without having to be directly programmed. Machine learning is concerned with developing computer programmes that can access data and learn on their own. The introduction of the internet has revolutionised the use of machine learning in today's century. The new scheme, which seeks to create a quantifiable trust assessment model, then measures specific confidence qualities numerically. An epic calculation based on AI standards is conceived to portray the separated certainty highlights and join them to deliver a last trust characteristic to be utilized for dynamic. One of the methods used is the Generic Trust Computational Model (GTCM). It's a prototype that displays relevant details about the confidence acquisition and evaluation process using three Trust Metrics (TMs): experience, practise, and reputation. The Machine Learning Model uses the Principal Component Analysis (PCA) calculation, which depends on Singular Value Decomposition (SVD), to lessen the $\mathrm{N}$ measurements to two for perception purposes. In a number of implementations, Independent Component Analysis (ICA) has outperformed standard PCA. When Principal Component Analysis failed to differentiate eye artefacts from brain signals, particularly when their amplitudes were identical, it was used to exclude them from the ElectroEncephaloGram (EEG).

Keywords : AI, Machine learning, Independent component Analysis (ICA),BSS, PCA, EEG, ECG.

\section{INTRODUCTION}

Independent Component Analysis (ICA) is a machine learning tool for differentiating between independent and mixed signals. Singularity is pushed in Autonomous Variable Investigation. The Free Part Examination calculation partitions the sign into subcomponents that are added substance. This is accomplished by accepting that the subcomponents are non-Gaussian signs that are measurably free of one another. Blind Source Separation (BSS) is an issue in which signals are separated exclusively on the basis of their mixture. Without needing to access the movements directly, the mixing system is conscious 
of them. In most cases, only the sources general statistical and structural properties, as well as the mixing mechanism, are required. Assume that no training data is allowedto be transmitted due to bandwidth limitations. The reception will be held in full darkness in that situation. BSS tends to be and continues to be a difficult challenge, particularly in its broadest context. It's worth noting that, on average, humans have no difficulties coping with the cocktail party catastrophe. This fact should not be overstated, though, due to the remarkable complexity and performance of two human neural networks. If relying solely on the recordings of a few microphones located around the venue, it will be difficult to separate speakers who are conversing at the same time. Nonetheless, much improvement has been made in the study of the BSS dilemma in the last ten years or so. A significant number of successful general and specialised methods have been developed, and their applicability in a variety of settings has been demonstrated. As can be seen in this project, self-organizing neural networks have played a significant role in this study, exploiting human capacities for BSS by comparing them to analogous biological mechanisms. Overview of the major aspects of the BSS problem as well as potential solutions. For purposes that will be addressed, only methods that rely on higher-order statistics are discussed. In addition to well-known and wellestablished results and algorithms, many more recent advancements are explored that show potential in improving/extending the capabilities of older, conventional approaches and removing their shortcomings. Face vibrations, eye flickers, body clamor, heart signals, and line commotion are on the whole basic wellsprings of critical and diverting relics in electroencephalographic (EEG) chronicles. The improved calculation recognizes wellsprings of superGaussian and sub-Gaussian sufficiency appropriations, permitting sub-Gaussian line commotion to be dense utilizing a solitary source channel got from the information. Possibilities from various pieces of the mind, skin, and body are directly added at the anodes. There are almost no transmission pauses. Since it utilizes spatial channels got from the Free Part Aanalysis calculation, the strategy doesn't have reference channels for every antiquity source. By deleting the inputs of the artifactual sources, "corrected" EEG signals can be derived after collecting the individual time courses of different brain and artefact sources from the results. The Independent Component Analysis algorithm can detect $\mathrm{N}$ sources if there are $\mathrm{N}$ sensors. On account of EEG signals, accept that multichannel EEG chronicles are combinations of the basic cerebrum and artifactual signs. Since volume conduction is thought to be straight and immediate, the assumption is satisfied. Since eye and muscle withdrawals, line commotion, and heart motivations are only occasionally time-bolted to EEG enactment locales, which are accepted to connote neuronal association in cortical neurons, the reason is objective. This conclusion is suspicious because don't know how many statistically independent impulses lead to the scalp EEG. Utilizing countless low-level and transiently unmistakable source enactments, factual computations have shown that the ICA calculation can precisely observe the actuation time courses and scalp geographies of moderately huge and transiently isolated sources from virtual scalp accounts. EEG behaviour is contaminated by face movements, blinks, muscle, pulse, and line noise, making it impossible to interpret and analyze. A variety of methods have been used to isolate face twitch and blink artefacts from EEG recordings:

A large amount of accumulated data is lost as contaminated EEG epochs are rejected. Noise channels with no clear reference pathways, such as muscle noise, electrodenoise, and line noise, cannot be eliminated using regression techniques. 
By removing inputs from artifactual sources onto the scalp sensors, A wide range of artifacts can be extracted from multichannel EEG files using ICA. Our findings show that ICA can map, remove, and erase behaviour in EEG documentation from a variety of artifactual sources with comparable outcomes to regression-based and Principal Component Analysis approaches. ICA-based antique remedy can separate and eliminate a wide assortment of curios from EEG information utilizing straight disintegration.

\section{RELATED STUDY}

Blind Source Partition (BSP) and Independent segment examination (ICA) are all things considered subject to a wide class of independent learning counts. Discovered future applications in a wide range of areas, from desire to neuroscience. Another model in BSS is to consider issues in the course of action of cross area factorization or all the more wide signs rot with probabilistic generative and tree-facilitated graphical models and experience closed information about authentic substance and plan of lethargic factors or sources, for example, Spatio-transient decorrelation, quantifiable freedom, little condition, perfection or most lessened complex nature one may say, e. g. , of best consistency.

The attainable objective of such disintegrating can be considered as the examination of sources over the top truly free and cutoff points of a blending framework or significantly more by and large as tracking down another diminished or reformist and facilitated portrayal for the saw (sensor) information that can be unwound as really basic coding or apparently impeded source assessment. The focal matter of debate is to discover such change or coding (straight or nonlinear) which has genuine importance and understanding. present a BSS and ICA audit of different metrics for static and dynamic models, as well as their implementations. The paper basically consolidates three bits: (1) BSS figuring for static models (brief mixes); (2) increase of BSS and ICA converging with little condition or non-investigation objectives; (3) BSS implies dynamic models (convolutive mixes).

Independent part examination (ICA) is a class of estimations by and large applied to detach sources in EEG data. Most ICA approaches use progression rules got from common verifiable opportunity and are invariant concerning particular insights' certifiable mentioning. Propose arranging genuine signs in a perplexing vector space that considers earthly sign requests and adheres to such mixing stationarity imperatives. The following strategy, which we call Pairwise Complex Free Part Assessment (PWC-ICA), plays out the ICA in a stunning setting and a brief timeframe later revaluates the essential understanding space results. Examine the presentation of our new solution in comparison to a few current ICA measurements for the visually impaired source detachment Blind Source Separation problem using true and recreated EEG data.

\section{PROPOSED SYSTEM}

Throughout the system, a never-ending jumble of signals from various origins happens. The colours in the below diagram blend together to create new shades and tones. All we see is the product of the merger of simpler elements. Are largely unaware that the world around us is a jumble of autonomous processes; only become mindful of the uncertainty as many signals that do not mix fight for our attention. A cocktail party scenario where one is listening to another person's voice while tuning out the sounds of the other guests is a good example. Depending on the level of noise in the room, this may be a simple or difficult process. Despite this, our brains can tell the difference between signal and noise. Any statistical 
approach attempts to break down a signal into its component bits, but it's mysterious how our brains do it. One of these approaches is Independent Component Analysis.

Blind Source Separation, also known as Signal-Space Separation, is a physics-based technique for separating sources. Signal-Space Separation separates the measured signal into components attributed to sources within the sensor array's measurement volume (internal components) and external components attributed to sources outside the measurement volume (the external components). The external components may be eliminated to minimise ambient noise when the internal and external components are linearly separated. The haemodynamic reaction has a frequency content of less than $0.5 \mathrm{~Hz}$. There is an increase in activity at 1 $\mathrm{Hz}$. Since it is needless, we use a low pass filter to block the person's heartbeat. To remove data drift, a high pass filter is often used.

Brain signals can be extracted using EEG. EEG signal information may be pre-processed to create a bandpass filter. The ICA method is used to separate the signals. Independent Component Analysis can be decoded into image execrations that can show in Figure 1, which can then be further analysed to generate function source signals. They separate the signals from the unit and projection as a whole.

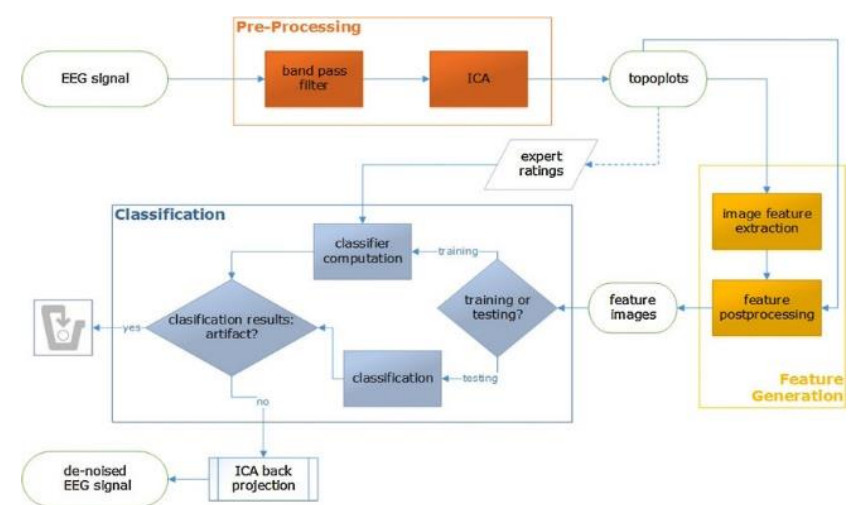

Figure 1 Architecture for Independent component Analysis.
This includes the fact that there are several alternatives, source separation algorithms. The ideas can be summarised using four different approaches.

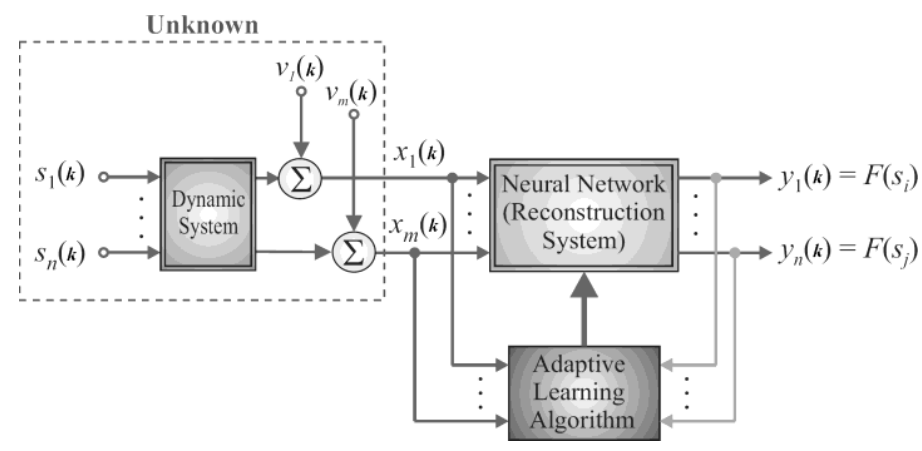

Fig 2(a)General model for Blind Source Separation

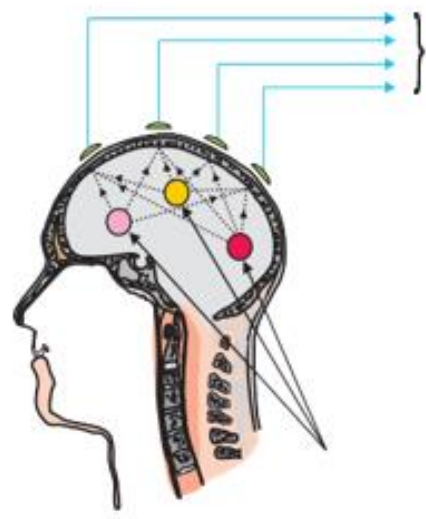

Fig 2(b)Brain Activity using EEG or MEG Dipolar brain sources

Fig 2(a) General model for Dazzle Source Separation Fig 2(b)Brain Movement utilizing EEG or MEG

Figure 2(a) General model representing blind source partition (BSS), 2(b) Such models are misused in nonobtrusive multi-sensor recording of cerebrum movement utilizing EEG or MEG. Superposition neural brain signals and non-brain origins connected to eye and muscle movements are believed to be picked up by scalp receptors (electrodes, squids).

As the expense work, the most famous arrangement utilizes a measurement of sign factual freedom, nonGaussianity, or scantiness. At the point when sources are thought to be measurably free yet come up short on a transient construction, Higher-Request 
Measurements (HOS) are important to take care of the BSS issue (verifiably or unequivocally). For this situation, the convention doesn't take into consideration more than one Gaussian source.

\section{Proposed Algorithm}

A sine wave with a fixed frequency can be used in all of the sources. Anything blends depending on your viewing point. This implies that in a mixed-signal, the source nearest to you is more effective than the one further away.

$$
x=A s
$$

By multiplying $\mathrm{x}$ with the inverse of $\mathrm{A}$, can recover the source signals from the mixed signals, according to our model:

$$
\begin{gathered}
W=A^{-1} \\
s=x W
\end{gathered}
$$

This necessitates locating the root signals and quantifying $\mathrm{W}$.

$$
\operatorname{covariance}(\check{X})=I
$$

Where I is the identity matrix.

In our implementation of ICA, however, we will not use kurtosis as a contrast element. We'll do it again later to double-check our findings. Instead, we can use the $\mathrm{g}(\mathrm{u})$ contrast function and its first derivative, $g^{\prime}(\mathrm{u})$ :

$$
\begin{aligned}
& g(u)=\tanh (u) \\
& g^{\prime}(u)=1-\tanh ^{2}(u)
\end{aligned}
$$

The FastICA algorithm uses two above functions in a fixed-point iteration scheme as follows:

$$
\begin{aligned}
& \text { for } 1 \text { to number of components } c \text { : } \\
& w_{p} \equiv \text { random initialisation } \\
& \text { while } w_{p} \text { not }<\text { threshold: } \\
& w_{p} \equiv \frac{1}{n}\left(X g\left(W^{T} X\right)-g^{\prime}\left(W^{T} X\right) W\right) \\
& w_{p} \equiv w_{p}-\sum_{j=1}^{p-1}\left(w_{p}^{T} w_{j}\right) w_{j} \\
& w_{p} \equiv w_{p} /\left\|w_{p}\right\| \\
& W \equiv\left[w_{1}, \ldots, w_{c}\right]
\end{aligned}
$$

\section{EXPERIMENTAL RESULTS}

Consider the properties of our source signals when using the ICA to correctly approximate W. For the algorithm to work, the mixed signals must first be a linear combination of a variety of source signals. As the second precondition, the source signals must be self-contained. What does it entail to be selfsufficient? Signal s1 doesn't know anything about signal s2, and signal s2 doesn't know anything about signal s1. This may indicate that they are uncorrelated, with a zero covariance. As the third precondition, the individual components must be non-Gaussian. The density distribution of two nongaussian signals paired on a cube will be uniform; see the upper left plot in the Figure 3 below. When these two signals are combined in an orthogonal matrix, they no longer exist as separate signals and have a uniform distribution on a parallelogram (see lower left plots).
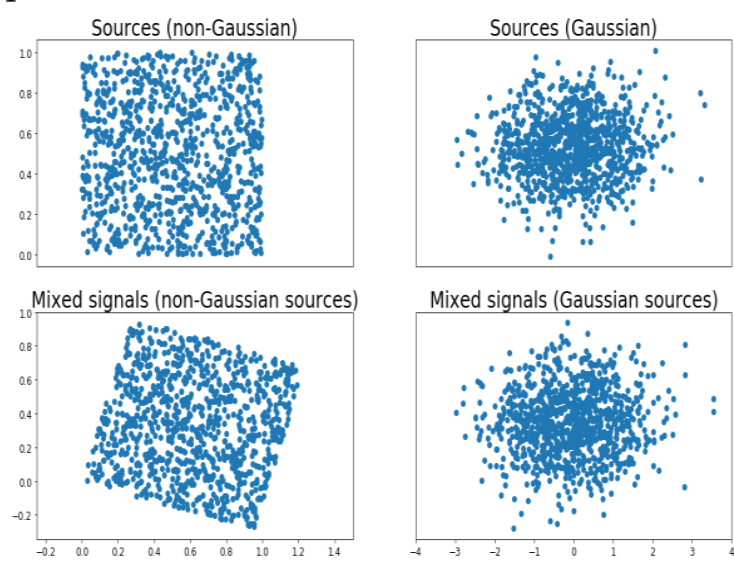

Fig 3: Comparison of Gaussian and non-Gaussian Signals 
So, let's see if our earlier test signals satisfy these specifications. In the left plot below, the sine wave signal is plotted against the saw-tooth signal, with the colour of each dot representing the noise portion. The signals are distributed in a rectangle, as expected for non-Gaussian random variable. The ICA's results are shown in the figure 4 below. The root signals are shown in the upper panel, while the individual components obtained by our ICA implementation are shown in the lower panel. And the end result is fantastic. Our appeal was met by all three networks.

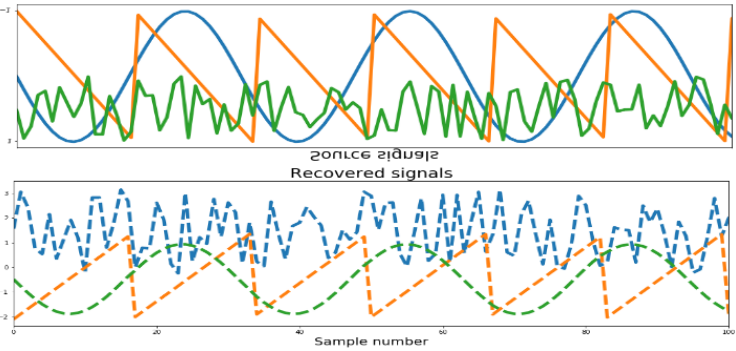

Fig 4 :Source signaland Recovered signal from ICA

Finally, let's take a look at signal kurtosis. As can be shown figure 5 , both of our mixed messages have a kurtosis of one. In the other hand, both recovered independent elements have a kurtosis of 1. 5, suggesting that are less Gaussian than their origin. This must be the case since the ICA aims to maximise non-Gaussianity. It clearly demonstrates that, as previously mentioned, the mixture of non-Gaussian signals would be more Gaussian than the source.

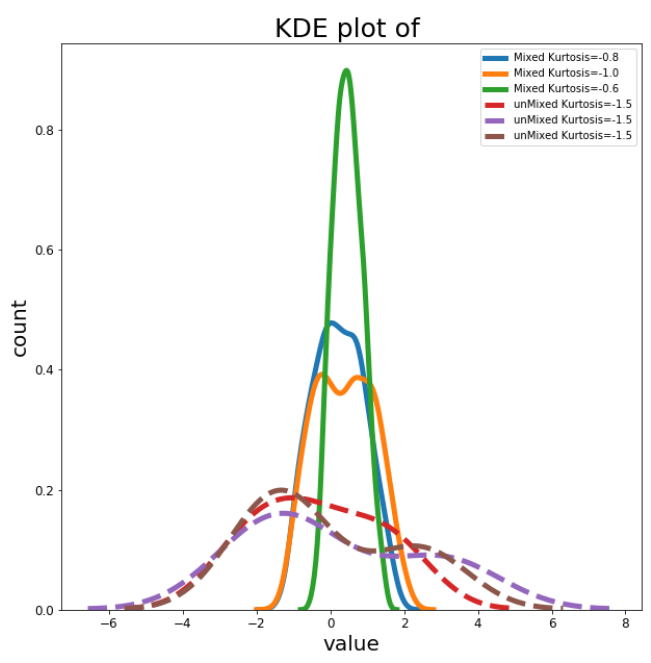

Fig 5 Kurtosis of the signals.
The haemodynamic reaction has a frequency content that is mainly below $0.5 \mathrm{~Hz}$. The data indicates a 1 $\mathrm{Hz}$ increase in movement, which is unwelcome and is triggered by the person's heartbeat. A low pass filter is used to prevent this. A high pass filter is used to remove slow drifts in the data shown in figure 6.
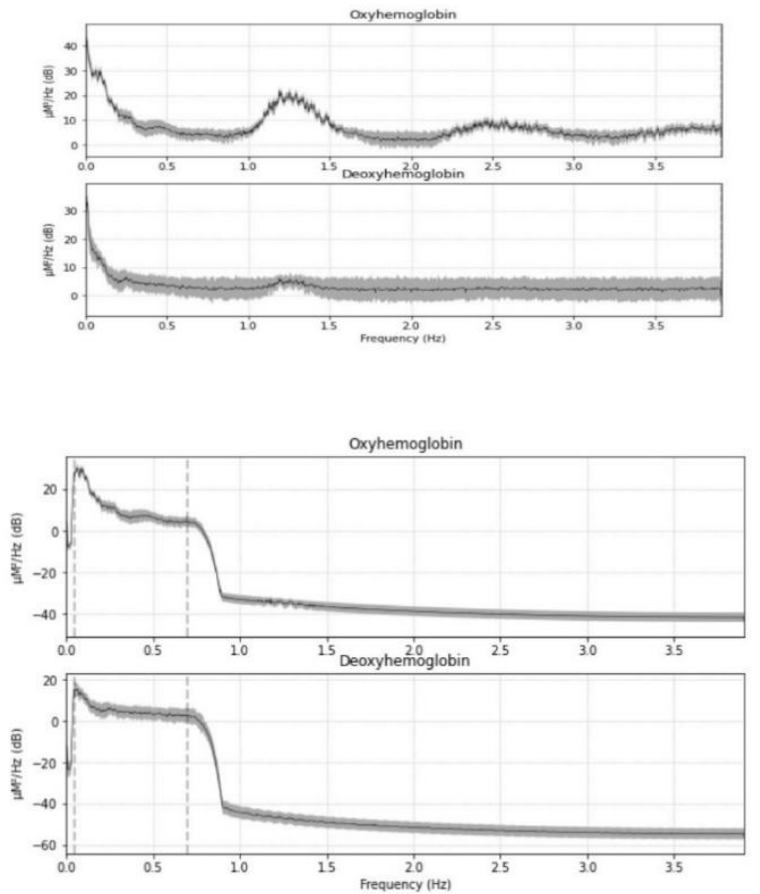

Fig 6 Before and After Removing heart rate from Signal.

Build the most widely used fNIRS data visualisation as shown in figure $7, \mathrm{HbO}$ and $\mathrm{HbR}$ are plotted on the same graph to illustrate how the two signals communicate.

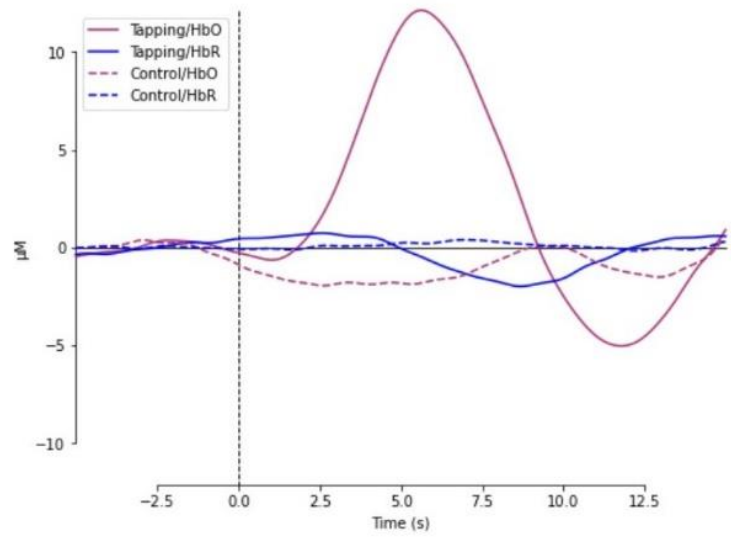

Fig 7 Two signals Response Graph.

V. CONCLUSIONS AND FUTURE WORK 
Independent Component Analysis separates a multivariate sign into subcomponents. Blind signal is kinds of ICA. Source signal are the yield of mind signals. We can take the source signals and concentrate the vital data through ICA. The vital data is only, what we see, what we hear and so on.

In Future work, new based features on Linear Discriminant analysis can be applied. When measurements on independent variables on each observation are continuous quantities, LDA works. Discriminant correspondence analysis is the same approach when dealing with categorical independent variables.

\section{REFERENCES}

[1]. Nunez, P. L. (1981) Electric Fields of the Brain (Oxford Univ. Press, New York).

[2]. Scherg, M. \& Von Cramon, D. (1986) Electroencephalogr. Clin. Neurophysiol. 65, 344360.

[3]. Dale, A. M. \&Sereno, M. I. (1993) J. Cogn. Neurosci. 5, 162-176.

[4]. Chapman, R. M. \& McCrary, J. W. (1995) Brain Lang. 27, 288-301.

[5]. Bell, A. J. \&Sejnowski, T. J. (1995) Neural Comput. 7, 1129-1159.

[6]. Snyder, A. Z., Abdullaev, Y. G., Posner, M. I. \&Raichle, M. E. (1995) Proc. Natl. Acad. Sci. USA 92, 1689-1693.

[7]. Hillyard, S. A. \&Munte, T. F. (1984) Percept. Psychophys. 36, 185-198.

[8]. Makeig, S., Bell, A. J., Jung, T.-P. \&Sejnowski, T. J. (1996) Advances in Neural Information Processing Systems 8, 145-151.

[9]. S. Takahashi, Y. Anzai, and Y. Sakurai, "A new approach to spike sorting for multi-neuronal activities recorded with a tetrode- how ICA can be practical," Neuroscience Research, vol. 46, no. 3, pp. 265-272, 2003.

[10]. A. Tonazzini, E. Salerno, and L. Bedini, "Fast correction of bleed-through distortion in grayscale documents by a blind source separation technique," International Journal on Document Analysis and Recognition, vol. 10, no. 1, pp. 17-25, 2007.

[11]. T. Ristaniemi and J. Joutsensalo, "Learning algorithms for blind multiuser detection in CDMA downlink," in Proceedings of the 9th IEEE International Symposium on Personal, Indoor and Mobile Radio Communications, vol. 3, pp. 10401044, September 1998.

[12]. L. H. Sibul, M. J. Roan, and J. Erling, "Deconvolution and signal extraction in geophysics and acoustics," The Journal of the Acoustical Society of America, vol. 112, no. 5, p. 2389, 2002.

[13]. L. T. Duarte, R. Lopes, J. H. Faccipieri et al., "Separation of reflection from diffraction events via the CRS technique and a blind source separation method based on sparsity maximization," in Proceedings of the 13th International Congress of the Brazilian Geophysical Society, 2013.

[14]. B. Rivet, L. Girin, and C. Jutten, "Mixing audiovisual speech processing and blind source separation for the extraction of speech signals from convolutive mixtures," IEEE Transactions on Audio, Speech and Language Processing, vol. 15, no. 1-4, pp. 96-108, 2007. 16H.-M. Park, H.-Y. Jung, T.-W. Lee, and S.-Y. Lee, "Subbandbased blind signal separation for noisy speech recognition," Electronics Letters, vol. 35, no. 23, pp. 2011-2012, 1999.

[15]. A. J. Bell and T. J. Sejnowski, "An informationmaximization approach to blind separation and blind deconvolution," Neural Computation, vol. 7, no. 6, pp. 1129-1159, 1995.

[16]. T.-W. Lee, M. Girolami, and T. J. Sejnowski, "Independent component analysis using an extended infomax algorithm for mixed subgaussian and supergaussian sources," Neural Computation, vol. 11, no. 2, pp. 417-441, 1999.

[17]. J. Karvanen, J. Eriksson, and V. Koivunen, "Pearson system based method for blind separation," in Proceedings of 2nd International Workshop on Independent Component Analysis and Blind Signal Separation (ICA '00), pp. 585-590, Helsinki, Finland, June 2000. 20J. Karvanen and V. Koivunen, "Blind separation methods based on Pearson system 
and its extensions," Signal Processing, vol. 82, no. 4, pp. 663-673, 2002.

[18]. A. Hyvarinen, "Fast and robust fixed-point algorithms for " independent component analysis," IEEE Transactions on Neural Networks, vol. 10, no. 3, pp. 626-634, 1999.

[19]. S. Amari and A. Cichocki, "Adaptive blind signal processing-Neural network approaches," Proc. IEEE, vol. 86, pp. 2026-2048, Oct. 1998.

[20]. A. K. Barros, A. Mansour, and N. Ohnishi, "Adaptive blind elimination of artifacts in ECG signals," in Proc. 1998 Workshop on Independence and Artificial Neural Networks, Tenerife, Spain, 1998, pp. 1380-1386.

[21]. A. K. Barros, R. Vigário, V. Jousmäki, and N. Ohnishi, "Extraction of periodic signals from multichannel bioelectrical measurements," IEEE Trans. Biomed. Eng., vol. 47, pp. 583-588, May 2000.

[22]. A. Bell and T. Sejnowski, "An informationmaximization approach to blind separation and blind deconvolution," Neural Computation, vol. 7, pp. 1129-1159, 1995.

[23]. S. Blanco, H. Garcia, R. Q. Quiroga, L. Romanelli, and O. A. Rosso, "Stationarity of the EEG series," IEEE Eng. Med. Biol., pp. 395-399, 1995.

[24]. J.-F. Cardoso, "Source separation using higher oerder moments," in Proc. 1989 IEEE Int. Conf. Acoustics, Speech, and Signal Processing (ICASSP'89), Glasgow, Scotland, 1989, pp. 21092112.

[25]. Blind signal separation: Statistical principles," Proc. IEEE, vol. 86, pp. 2009-2025, Oct. 1998.

[26]. High-order contrasts for independent component analysis," Neural Computation, vol. 11, pp. 157-192, 1999.

[27]. P. Comon, "Independent component analysis-A new concept?," Signal Processing, vol. 36, pp. 287314, 1994.

[28]. M. Hämäläinen, R. Hari, R. Ilmoniemi, J. Knuutila, and O. V. Lounasmaa, "MagnetoencephalographyTheory, instrumentation, and applications to noninvasive studies of the working human brain," Rev. Modern Phys., vol. 65, no. 2, pp. 413-497, 1993
[29]. H.-S. Fang, J. Cao, Y.-W. Tai, and C. Lu. Pairwise body-part attention for recognizing human-object interactions. In ECCV, 2018.

[30]. V. Delaitre, I. Laptev, and J. Sivic. Recognizing human actions in still images: a study of bag-offeatures and part-based representations. In BMVC, 2010.

[31]. Y. W. Chao, Z. Wang, Y. He, J. Wang, and J. Deng. Hico: A benchmark for recognizing human-object interactions in images. In ICCV, 2015.

[32]. C.-Y. Chen and K. Grauman. Predicting the location of "interactees" in novel human-object interactions. In ACCV, 2014.

[33]. A. Mallya and S. Lazebnik. Learning models for actions and person-object interactions with transfer to question answering. In ECCV, 2016.

\section{Cite this article as :}

N NITHYANANDAM "Independent Component Analysis for IoT Services Using Machine Learning", International Journal of Scientific Research in Computer Science, Engineering and Information Technology (IJSRCSEIT), ISSN : 2456-3307, Volume 7 Issue 2, pp. 309-316, MarchApril 2021. Available at doi : https://doi.org/10.32628/CSEIT217264 Journal URL : https://ijsrcseit.com/CSEIT217264 